\title{
Evaluation of root and canal morphology of maxillary permanent first molars in an Emirati population; a cone-beam computed tomography study
}

Eman Al Mheiri ${ }^{1,2}$, Jahanzeb Chaudhry ${ }^{1}$, Salma Abdo ${ }^{3,4}$, Rashid El Abed ${ }^{1}$, Amar Hasan Khamis ${ }^{1}$ and Mohamed Jamal ${ }^{1 *}$ (D)

\begin{abstract}
Background: The aim of this study was to analyze the root and canal morphology of the maxillary permanent first molars in an Emirati population using cone-beam computed tomography (CBCT).

Methods: Two hundred and sixty-one CBCT scans were acquired. The data were extracted and anonymized to remove all patient identifiers. Two observers (an endodontic resident and an endodontist) evaluated all scans on diagnostic quality monitors.

Results: The prevalence of a second mesiobuccal canal (MB2) was $80.1 \%$ in all examined samples. Type II Vertucci classification, was the most common canal configuration (59\%) in the mesiobuccal root, followed by Types I (19.9\%) and IV (15.3\%), while Type III was the least common (5.7\%). Types I, II, and IV were significantly more common in the 21-40-year age group $(P<0.001)$, while Type III was observed significantly more often in the $<20$-year age group $(P<0.001)$. No significant effect of gender on the prevalence of Vertucci classification in the mesiobuccal root of maxillary first molars $(P=0.74)$ was found. Analysis of bilateral symmetry showed that $80 \%$ teeth had perfect bilateral symmetry, whereas 20\% were asymmetrical. Type II canal configuration showed the highest prevalence of bilateral symmetry (48.7\%), followed by Type I (15\%) and Type IV (10\%), while Type III showed the least prevalence of symmetry (3\%).

Conclusions: This was the first study to analyze the prevalence of MB2 canal in an Emirati population. Our results indicate high prevalence of MB2 (80.1\%) and emphasize the importance of using advanced techniques to locate the MB2 canal.
\end{abstract}

Keywords: Emirati population, Second mesiobuccal canal, Canal configuration, Cone-beam computed tomography

\footnotetext{
* Correspondence: mohamed.jamal@mbru.ac.ae

'Department of Endodontics, Hamdan Bin Mohammed College of Dental

Medicine, Mohammed Bin Rashid University of Medicine and Health

Sciences, Building 14, Dubai Health Care City, Dubai, United Arab

EmiratesP.O.Box: 505055

Full list of author information is available at the end of the article
}

C C The Author(s). 2020 Open Access This article is licensed under a Creative Commons Attribution 4.0 International License, which permits use, sharing, adaptation, distribution and reproduction in any medium or format, as long as you give appropriate credit to the original author(s) and the source, provide a link to the Creative Commons licence, and indicate if changes were made. The images or other third party material in this article are included in the article's Creative Commons licence, unless indicated otherwise in a credit line to the material. If material is not included in the article's Creative Commons licence and your intended use is not permitted by statutory regulation or exceeds the permitted use, you will need to obtain permission directly from the copyright holder. To view a copy of this licence, visit http://creativecommons.org/licenses/by/4.0/ The Creative Commons Public Domain Dedication waiver (http://creativecommons.org/publicdomain/zero/1.0/) applies to the data made available in this article, unless otherwise stated in a credit line to the data. 


\section{Background}

One of the most frequent causes of endodontic treatment failure is difficulty in identifying and treating all or part of root canal anatomy [1-4] . A missed canal might harbor necrotic tissues or microorganisms which can lead to persistent periapical pathology. The root and canal morphology of the maxillary first molars are frequently studied because of their complex anatomy and being one of the most common teeth to have root canal treatment $[1,5]$. It is generally accepted that most maxillary first molars have 3 roots and 4 canals; various studies have reported prevalence of second mesiobuccal canal (MB2) in the range of 52-93\% [1, 6-11]. Despite its high prevalence, MB2 is still difficult to find due to diffuse calcification, its narrowness and unusual location of the orifice. Missed MB2 is considered one of the most common cause of endodontic treatment failure of maxillary first molars. This has been emphasized since 1969, when Franklin et al. [1], concluded that locating MB2 increases the success of endodontic therapies in maxillary first molars. In a more recent, 5-years prospective clinical study, which included 5600 root canal-treated and retreated teeth, it was concluded that failure to locate and manage existing MB2 in maxillary first molars would detrimentally affect the prognosis of root canal treatment [5]. Several other studies, showed similar findings, with missed MB2 in maxillary first molars resulting in endodontic treatment failure [1214]. Despite the recent advances in available diagnostic tools to evaluate maxillary first molars canal anatomy and locate MB2, such as the dental operating microscope (DOM) and cone-beam computed tomography (CBCT), the basic knowledge of maxillary first molar morphology and the prevalence of MB2, its variations and the effects of ethnicity on such variations is essential.

Several methods such as tooth clearing and staining, radiological methods (intraoral radiographs and CBCT), grinding and sectioning, and clinical observations [14, 15] have been used to study root and canal morphologies. CBCT allows the three-dimensional assessment of dental and maxillofacial structures by providing an excellent noninvasive model and hence, is indispensable for the analysis of root and canal anatomies [10, 16, 17]. Several studies have successfully used CBCT to analyze root and canal anatomies of the maxillary molars in different populations and ethnic groups with reported prevalence of MB2 in the range of $40-80 \%[10,18-25]$. After an extensive review of the literature, we determined that no study has analyzed the anatomy of maxillary first molars in the Emirati population. Such study if available will provide valuable information on possible variations of canal anatomy in maxillary first molars and prevalence of MB2 for dentists treating this population. Such information will aid in the decisionmaking process before, during and after endodontic treatment. It will also encourage dentists to use advanced technologies such DOM and CBCT or to refer to specialists if necessary. Therefore, the aim of this study is to describe the root and canal morphology of maxillary permanent first molars in an Emirati population using CBCT.

\section{Methods \\ Sample collection}

The institutional review board approvals were obtained from the concerned committees of the Mohammed Bin Rashid University of Medicine and Health Sciences and Abu Dhabi Health Authority to conduct this retrospective study. CBCT scans of patients who were treated at a community dental center between 2017 and 2018 were obtained and analyzed. The CBCT scans were acquired using the Planmeca ProMax CBCT scanner (Planmeca Oy, Helsinki, Finland). The imaging protocol was as follows: Field of view $($ FOV $)=16 \times 11 \mathrm{~cm}$; tube peak potential $=120 \mathrm{kVp}$; tube current $=18.54 \mathrm{~mA}$; time $=8.9 \mathrm{~s}$; voxel size $=0.4 \mathrm{~mm}$. The scans of patients who met the following inclusion criteria were included in the study: Emirati, age range $12-75$ years, presence of bilateral permanent maxillary first molars, completely matured and erupted teeth. Permanent maxillary first molars with root canal fillings, posts, crowns, coronal or root resorption, extensive coronal or root caries, and/or periapical or periradicular radiolucency were excluded from the study. Two hundred sixty-one scans that met the inclusion and exclusion criteria were randomly selected, anonymized, and exported from the Abu Dhabi Health Authority database in Digital Imaging and Communication in Medicine (DICOM) format. The sample size of 261 scans was determined based on power analysis using Cochran's test, where the number of MB2 in previous studies $[26,27]$ was considered the relevant difference.

\section{Radiographic evaluation}

Two observers (an endodontic resident and an expert endodontist) evaluated all scans on an iMAC computer ([27-in. screen size with Retina $5 \mathrm{~K}$ display, $5120 \times 2880$ resolution with support for 1 billion colors, 500 nits brightness], Apple, USA) in a room with controlled lighting using the Horos DICOM viewer (horosproject. org) [28]. The root and canal morphology were assessed using the three-dimensional multiplanar reconstruction (3D MPR) tool, in which all images were examined in the axial, coronal, and sagittal planes. Furthermore, the observers determined the coronal section to be within 2 $\mathrm{mm}$ of cemento-enamel junction (CEJ), middle third section to be within $2 \mathrm{~mm}$ of mid root length (from apex to $\mathrm{CEJ}$ ) and apical section to be at apex and $2 \mathrm{~mm}$ above. The two observers were trained and calibrated before the evaluation process. For observer training and calibration, a sample of CBCT scans, exhibiting all 8 types of root canal morphology in the maxillary first molars as per Vertucci classification (VC) [29], was used. 
The observers recorded the number of roots and the canal morphology of each root of the maxillary first molars as per VC (Table 1, Fig. 1). MB2 is considered to be present if one of the two canal configurations of $\mathrm{VC}$ (Type II, III, IV, V, VI and VII) is observed in the mesiobuccal root (Fig. 1). Moreover, the symmetry in canal morphology between the right and left sides was recorded. The observers reviewed and recorded the findings twice with a gap of 30 days between the two reviews. The sequence of scans in the second review was different from that in the first review. The findings were finalized based on recordings of both the observers. Disagreements between the observers were resolved by consulting a third evaluator (expert endodontist). The data were subjected to kappa test to ensure reproducibility and reliability, and the Altman's scale was used for interpretation. Finally, the findings were tabulated and were correlated with age and gender.

\section{Statistical analysis}

Data were analyzed using SPSS for Windows version 25.0 (SPSS Inc., Chicago, IL). Results were crosstabulated to examine the dependency between variables. Statistical analysis was performed using $x^{2}$ (Chi-square) to determine of association between variables such as distribution of MB2 by gender, age or site. Kappa test was used to test inter- and intra-rater reliability. Frequency tables' bar and lines graphs were used as descriptive statistics. A $P$-value of less than 0.05 was considered significant in all statistical analysis.

\section{Results}

As planned, the two observers reviewed the $261 \mathrm{CBCT}$ scans independently with focus on left and right maxillary first molars. As only CBCT scans of patients who have bilateral maxillary first molar were included, therefore a total of $522 \mathrm{M}$ were studied. The kappa test indicated moderate concordance within the same examiner $($ Kappa $=0.44)$ and good concordance among the 2 examiners $($ Kappa $=0.61)$. The expert opinion was required in 58 out of 261 scans.

Of the 261 patients, 145 (55.6\%) were females and 116 (44.4\%) were males. The age of patients ranged from 12 to 71 years; $28 \%$ were younger than 21 years, $52.1 \%$ were between 21 and 40 years and 19.1\% were older than 40 years (Table 2).

Among the studied 522 maxillary permanent fist molars, most teeth had 3 roots (98.9\%), while only 0.5 and $0.6 \%$ had 2 and 4 roots, respectively. Root canal configurations of only Types I and II of the VC were detected in the palatal and distobuccal roots, of which Type I was more prevalent, while Type II was observed in only 1.2 and $2 \%$ in the palatal and distobuccal roots, respectively (Table 3). The mesiobuccal root showed a single canal configuration (Type I) in 19.9\%, while the 2-canal configurations (Types II, III, or IV) were observed in $80.1 \%$. Most mesiobuccal roots had Type II VC (59\%), followed by Type I and Type IV (19.9 and 15.3\%, respectively). The least common canal configuration in the mesiobuccal root was Type III (6\%) (Table 3). No other types were observed in the mesiobuccal roots. Figure 2 shows an example of observed canal configurations in the mesiobuccal root.

Analysis of prevalence of a specific canal configuration in age groups in the mesiobuccal root showed that Types I, II, and IV were observed significantly more often in the 21-40-year age group than that among other age groups $(P<0.001)$. Type III was observed significantly more often in the $<20$-year age group than that in other age groups $(P<0.001)$. Further dependency analysis showed no significant effect of gender on canal configuration in the mesiobuccal root of maxillary first molars $(P=0.74)$.

The analysis of bilateral symmetry in canal configuration in the mesiobuccal root of right and left maxillary first molars showed that $80 \%$ canal configurations were bilaterally symmetrical and $20 \%$ were asymmetrical. More specifically, Type II had the highest incidence of bilateral symmetry (48.7\%), followed by Type I (15\%)

Table 1 Vertucci canal classification

\begin{tabular}{|c|c|}
\hline Type & Description \\
\hline Type I & A single canal extends from the pulp chamber to the apex \\
\hline Type $\|$ & Two separate canals leave the pulp chamber and join short of the apex to form a single canal \\
\hline Type III & One canal leaves the pulp chamber, divides into 2 within the root, and then merges to exit as a single canal \\
\hline Type IV & Two separate and distinct canals extend from the pulp chamber to the apex \\
\hline Type V & One canal leaves the pulp chamber and divides short of the apex into 2 separate and distinct canals with separate apical foramina \\
\hline Type VI & Two different canals go the pulp chamber, merge in the body of the root, and redevise short of the apex to exit as 2 distinct canals \\
\hline Type VII & $\begin{array}{l}\text { One canal leaves the pulp chamber, divides and then rejoins within the body of the root, and finally re-divides into } 2 \text { distinct canals short } \\
\text { of the apex }\end{array}$ \\
\hline $\begin{array}{l}\text { Type } \\
\text { VIII }\end{array}$ & Three separate and distinct canals extend from the pulp chamber to the apex \\
\hline
\end{tabular}




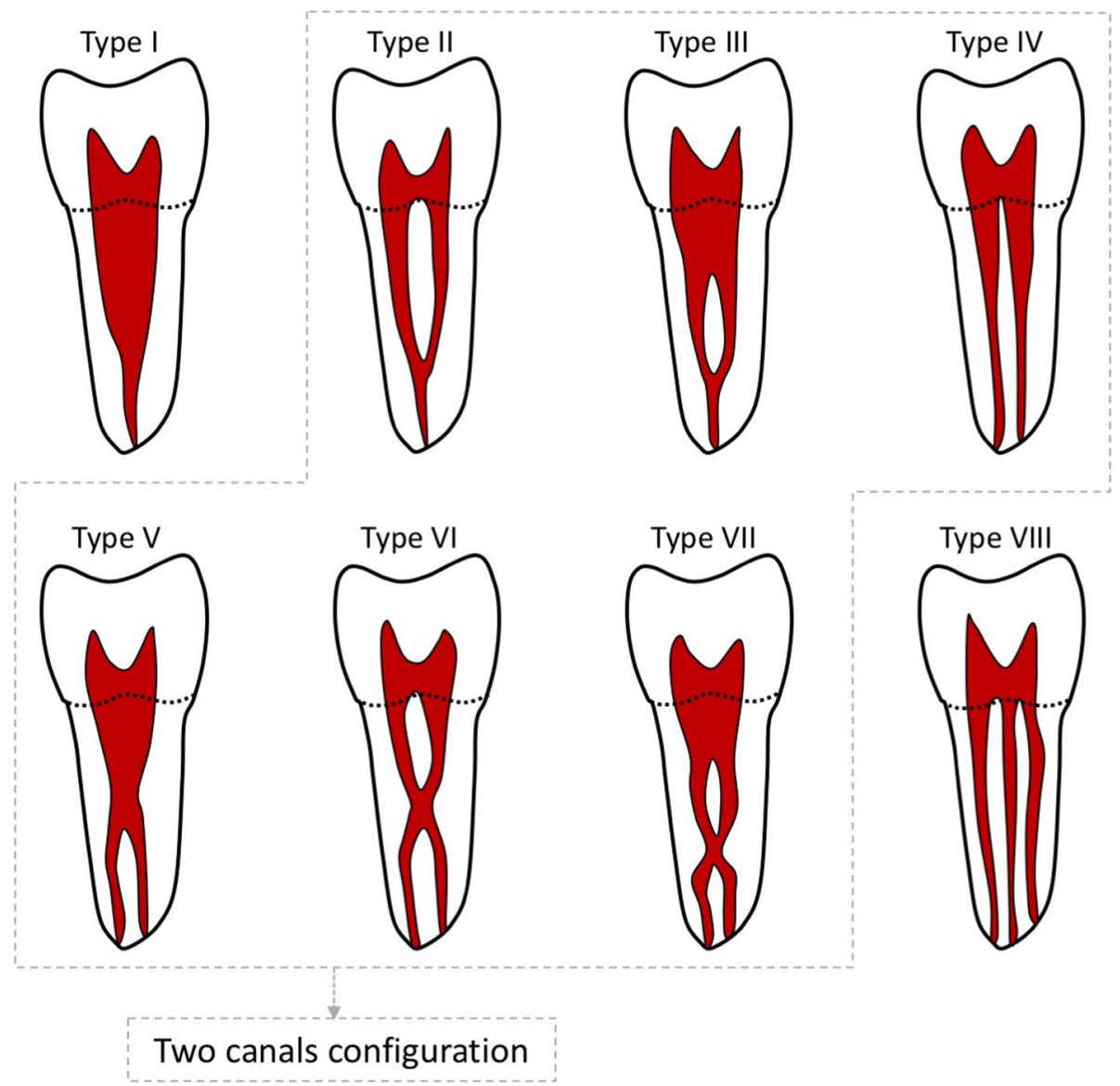

Fig. 1 Vertucci classification of the root canal system. Type II - Type VII (enclosed in gray dashed line) represent two canal configurations

and Type IV (10\%), while Type III had the least incidence (3\%) (Fig. 3).

\section{Discussion}

Endodontic treatment of the maxillary first molars is considered a challenge due to the complex root and canal anatomies and high prevalence of MB2 canal $[8,9$, 30]. Several studies have reported the importance of locating and cleaning the MB2 canal in the success of endodontic therapy $[4,5,30]$. Multiple approaches have been suggested to facilitate the localization of the MB2 canal and other anatomical variations, such as acquisition of knowledge regarding the differences in canal anatomy in different races and ethnic groups and using advanced clinical techniques such as DOM, stains, troughing with ultrasonic, and $\mathrm{CBCT}[9,16,31]$. Ball et al. in 2013 recommended the use of CBCT in cases

Table 2 Demographic Data

\begin{tabular}{lllll}
\hline Age & & \multicolumn{3}{c}{ Gender } \\
\hline$<20$ & $21-40$ & $>40$ & Female & Male \\
$73(28 \%)$ & $136(52.1 \%)$ & $52(19.9 \%)$ & $145(55.6 \%)$ & $116(44.4 \%)$ \\
\hline
\end{tabular}

with unexpected complex anatomy or with difficult to locate canals [31].

In this study we examined the root and canal configuration of the maxillary first molars in an Emirati population using CBCT. Our aim is to provide information to clinicians who treat this population and to address the knowledge gap related to the root and canal morphology of the maxillary first molars in this population. Our results showed that most teeth were 3 rooted (98.9\%) while only 0.4 and $0.6 \%$ had 2 and 4 roots respectively. Our results are similar to other studies which indicated that most maxillary first molars have 3 roots (range from

Table 3 Canal configuration per root of maxillary permanent first molar

\begin{tabular}{ccll}
\hline & Mesiobuccal root & Distobuccal root & Palatal root \\
\hline \multicolumn{2}{l}{ Single canal configuration } & & \\
Type I & $104(19.9 \%)$ & $512(98 \%)$ & $516(98.8 \%)$ \\
Two canals configuration & $10(2 \%)$ & $6(1.2 \%)$ \\
Type II & $308(59 \%)$ & - & - \\
Type III & $30(5.7 \%)$ & - & - \\
Type IV & $80(15.3 \%)$ & & \\
\hline
\end{tabular}




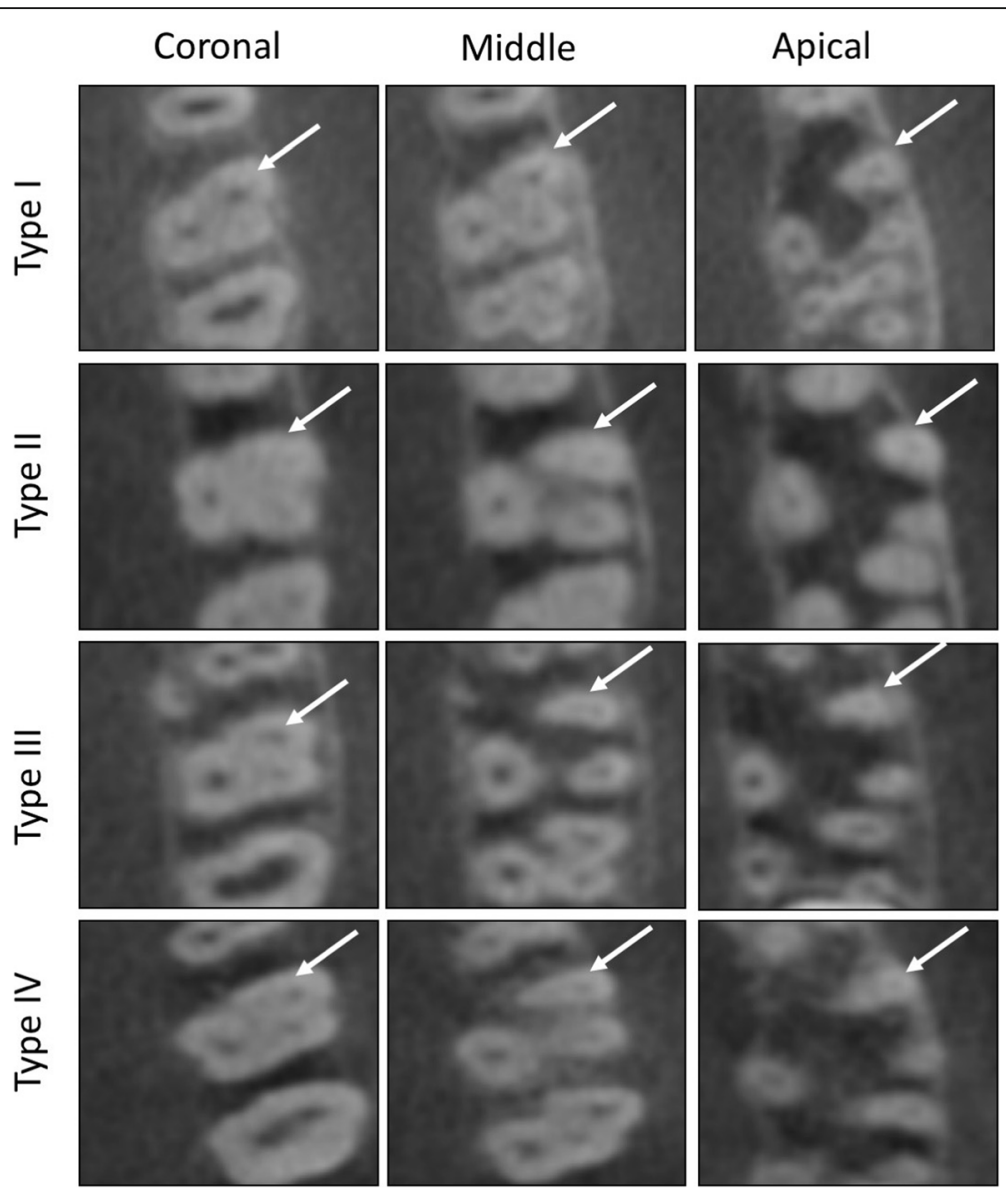

Fig. 2 Axial view of CBCT scans at different root levels showing the different canal configurations observed in the mesiobuccal root of maxillary permanent first molar. White arrows point to the mesiobuccal root

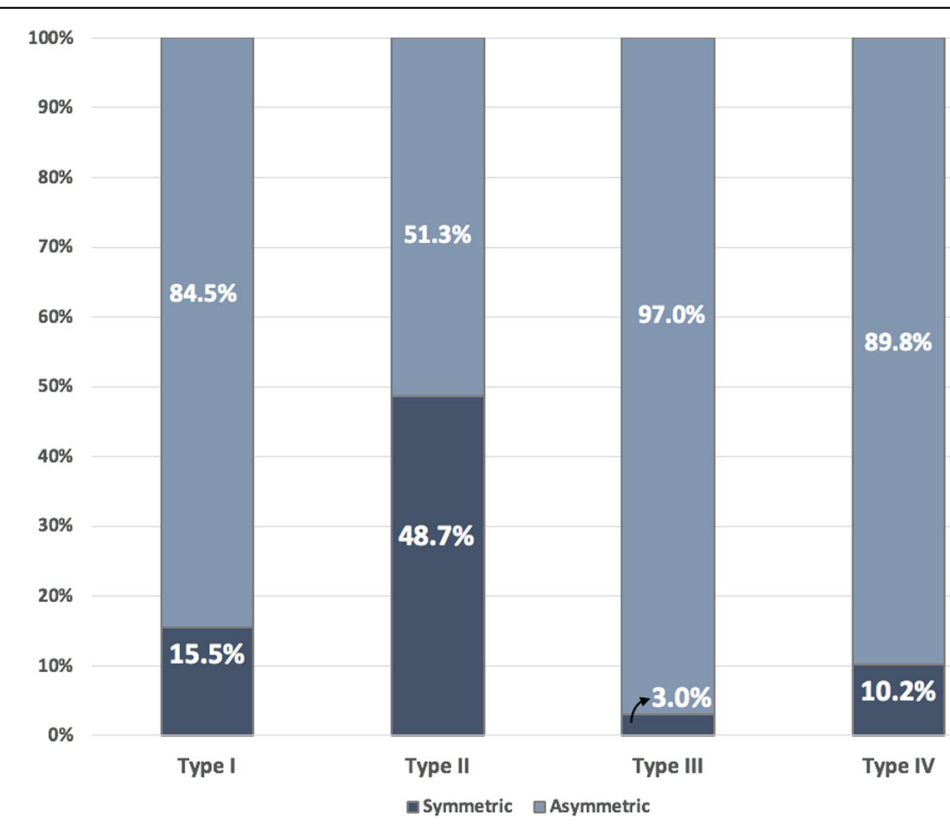

Fig. 3 Bar chart indicating the bilateral symmetry/asymmetry of different Vertucci classification in the mesiobuccal root 
82 to $100 \%$ ) followed by 2 roots (up to $9 \%$ ) $[6,10,11$, $18-27,32,33]$. Very few studies reported 4 rooted maxillary first molars. Neelakantan et al. reported 4 rooted maxillary first molars in $0.9 \%$ of examined teeth in Indian population [34]. Martin et al. studied the root and canal morphology of 5250 maxillary first molars using CBCT scans collected from 21 counties. They reported prevalence of 4 rooted maxillary first molar that range from 0 to $1.6 \%$ with an average of $0.2 \%$. Most examined molars in their study had 3 roots (94\%), followed by 2 roots $(5.4 \%)$ and 1 root $(0.4 \%)$ [35].

With regards to the canal morphology, our results showed that in the palatal and distobuccal roots, single canal configuration (Type I VC) was more prevalent, with only 1.2 and $2 \%$ of the examined teeth showed Type II VC in the palatal and distobuccal roots, respectively. These results are similar to several studies conducted in several populations, in which palatal and distobuccal root of maxillary first molars had single canal in a percentage range from 98 to $100 \%[6,11,23,25,27]$.

Our analysis for mesiobuccal root of examined maxillary first molars showed that $19.9 \%$ had single-canal configuration (Type I), while $80.1 \%$ showed 2-canal configurations (Type II, III, and IV VC). Our results are similar to those of several other studies conducted using CBCT in different populations worldwide. The prevalence of MB2 was reported to be $71 \%$ among the Portuguese population [18], $86.2 \%$ among the Spanish population [19], 88.5\% among the Brazilian population [20], 55.6\% among Saudi population [27], 40.3\% among the Italian population [21], $72.8 \%$ among the Egyptian population [22], 68.2\% among the American population [23], and 70.2\% among the Iranian population [24]. Kim et al. reported the prevalence of MB2 to be $64.6 \%$ among the Korean population [25], while Zhang et al. reported a prevalence of $52 \%$ among the Chinese subpopulation [10] (Fig. 4).

In our study, most maxillary first molars (59\%) exhibited the Type II VC. The prevalence of Type I and Type IV was 19.9 and $15.3 \%$, respectively. Our results are similar to those of Perez-Heredia et al., in which they found that Type II was the most common VC $(56.5 \%)$, followed by Type IV and Type I (23.2 and 13.8\%, respectively) [19]. Kim et al. reported that Type IV was the most common canal configuration (40.6\%), followed by Type I and II (36.4 and 20.4\%, respectively) [25]. Our results showed that Type III was the least common canal configuration (6\%). Moreover, Types V, VI, VII, and VIII VC were not observed. These results are in agreement with those of several similar CBCT studies, where Types VI, VII, and VIII were not observed in the mesiobuccal root of maxillary first molars [21, 22, 24, 27].

Our correlation analysis showed a significant relationship between age and the presence of MB2 canal. Types II and IV were observed significantly more often in the 21-40year age group than in the other age groups $(P<0.001)$, while Type III was observed significantly more often in the $<20$-year age group than in the other age groups $(P<$ $0.001)$. These results indicate that as age increases, the incidence of complex canal configuration decreases. Our results are in agreement with the findings of Reis et al., which indicated an inverse correlation between age and the presence of MB2 [20]. Similar findings were reported by Zheng et al., wherein MB2 was most commonly observed in the 20-30-year age group [11]. Neaverth et al. reported the highest incidence of MB2 in the 20-40-year age group [7]. This phenomenon could be attributed to the continuous dentin deposition over time resulting in canal calcification

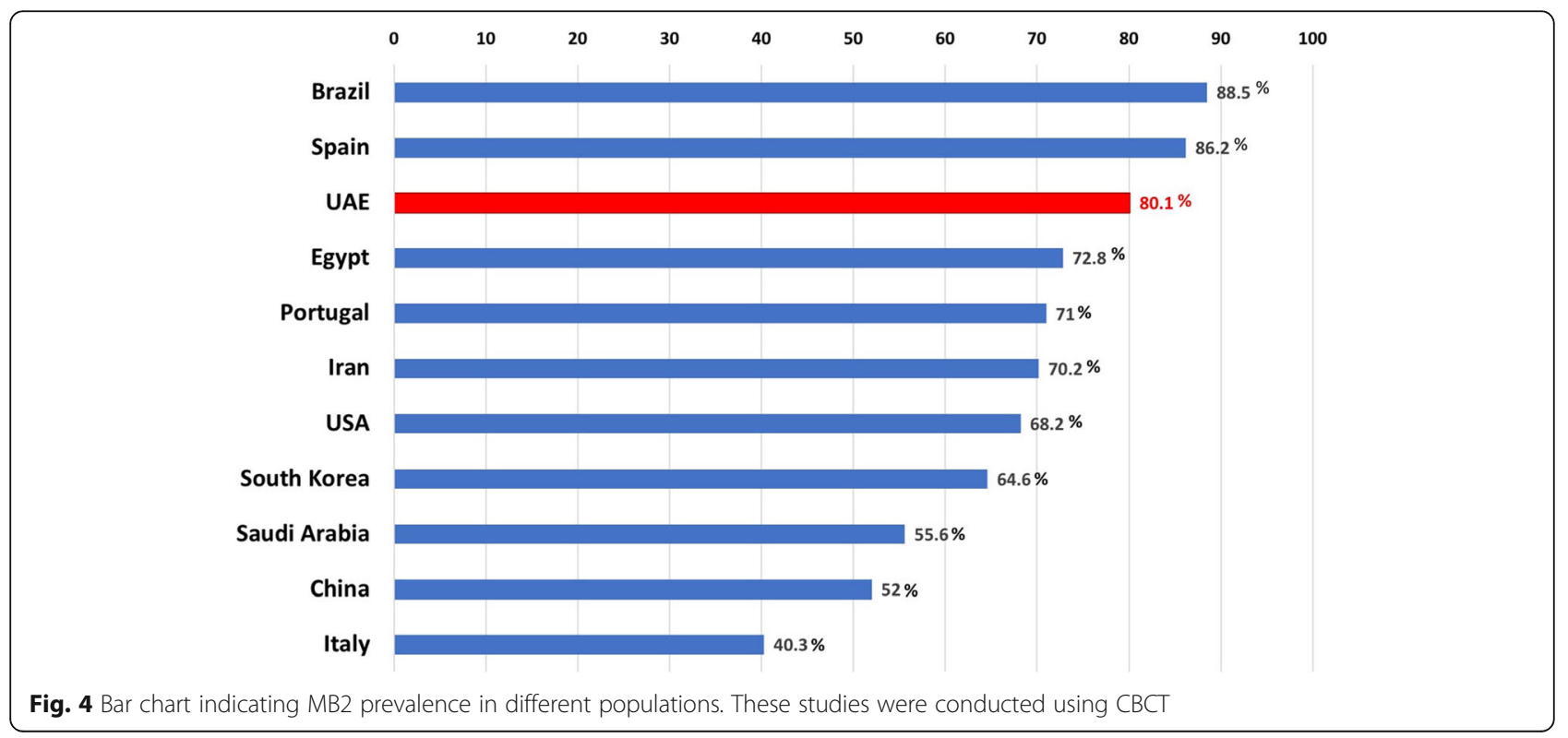


and narrowing. Using micro-CT scanning, Oi et al. reported a decrease in the size of the pulp cavity and in canal diameter with an increase in age [36]. Our correlation analysis showed no effect of gender on the presence of MB2 canal or a certain type of canal configuration in general.

The analysis of bilateral symmetry of canal configurations in the mesiobuccal root of right and left maxillary first molars showed that $80 \%$ canal configurations were bilaterally symmetrical and $20 \%$ were asymmetrical. More specifically, Type II VC showed the highest bilateral symmetry (48.7\%), followed by Type I (15\%) and Type IV (10\%), while Type III was the least prevalent (3\%). Our results are very similar to those of Plotino et al., which showed bilateral symmetry in $79.6 \%$ teeth [21]. Guo et al. reported bilateral symmetry in $65.6 \%$ teeth [23]. Interestingly, Type I VC showed 15\% symmetry implying that if a clinician finds a Type I VC (single canal configuration) on one side, there is an $85 \%$ chance that the other side would have any of the 2-canal configurations, Types II, III, or IV. Therefore, clinicians should continue their search for the MB2 canal on the other side, even if only one canal was encountered in the mesiobuccal root of the maxillary first molar on one side.

This study reported a relative high incidence of MB2 (80.1\%) in an Emirati population, and it was present in the form of type II, III and IV VC. Beside the importance of this information on encouraging clinicians to search and locate MB2, clinicians should be aware of the clinical challenges associated with the different forms of MB2 and plan their root canal treatment accordingly. For example, in case MB2 was in the form of type II $\mathrm{VC}$, then both canals in the mesiobuccal roots will share the same apical foramen, therefore there are high chances of iatrogenic cleaning and shaping errors, such as; over-enlarging the apical foramen, ledging, transportation and blockage of one of the canals [37]. Clinicians can avoid such errors by carefully selecting their cleaning and shaping protocol or clean and shape one of the canals to the full length while the other to the joining level [38, 39]. Another example are the clinical challenges related to MB2 in the form of type III VC, in which the MB2 orifice might not always be at the CEJ or pulp floor level but can start deeper apically. Therefore, clinicians should always scout the main canal with curved small hand file, especially if the orifice of MB2 was not found at the pulp floor level [37, 40, 41].

A possible limitation of this study is voxel size of CBCT scans, which was $0.4 \times 0.4 \mathrm{~mm}$. This voxel size results in lower image resolution than the image resolution obtained in comparable studies [18-20, 22, 23]. Such scans with lower resolutions could adversely affect an evaluator's ability to read the scans accurately, especially when reading small structures, such as calcified and lateral canals $[17,42]$. Bauman et al. showed that there is increase in detection rate of MB2 from 60.1 to $93.3 \%$ at voxel size of $0.4 \mathrm{~mm}$ to $0.125 \mathrm{~mm}$ [43]. Furthermore, Yan Ji et al. reported that even at $0.125 \mathrm{~mm}$ voxel size, it can still be challenging to detect small fine structure such as lateral canal [44].

\section{Conclusions}

Our study is the first to analyze the prevalence of MB2 canal in Emirati subpopulation. Our results show that the prevalence of MB2 in the Emirati subpopulation is relatively high (80.1\%). and emphasize the importance of searching for and using advance techniques to locate the MB2 canals in permanent maxillary first molars.

\section{Abbreviations}

3D MPR: Three-dimensional multiplanar reconstruction; CBCT: Cone-beam computed tomography; CEJ: Cemento-enamel junction; DICOM: Digital Imaging and Communication in Medicine; DOM: Dental operating microscope; MB2: Second mesiobuccal canal; VC: Vertucci classification

\section{Acknowledgments}

Not applicable.

\section{Authors' contributions}

E.M., Conceptualization, Methodology, Investigation, Data curation, Writing Original Draft; J.C. Methodology, Data curation, Writing - Review \& Editing; S.A. Resources, Writing - Review \& Editing; R.A. Investigation, Validation; A.K. Methodology, Formal analysis; M.J. Conceptualization, Methodology, Investigation, Writing- Reviewing and Editing. The authors read and approved the final manuscript.

Funding

This study received no study from profit or not-for-profit organizations.

\section{Availability of data and materials}

The datasets used and/or analysed during the current study are available from the corresponding author on reasonable request.

\section{Ethics approval and consent to participate}

The local Research Ethics Committee approved this study without restrictions under the protocol number: AAHEC-06-18-097. Through this approval the research team had the required administrative permissions to access the data used in this research. For this type of retrospective study, and as recommended by the Research and Ethics Committee, formal consent is not required.

\section{Consent for publication}

Not applicable.

\section{Competing interests}

The authors declare that they have no conflict of interest.

\section{Author details}

${ }^{1}$ Department of Endodontics, Hamdan Bin Mohammed College of Dental Medicine, Mohammed Bin Rashid University of Medicine and Health Sciences, Building 14, Dubai Health Care City, Dubai, United Arab EmiratesP.O.Box: 505055. ²Dental Department, Dubai Health Authority, Dubai, United Arab Emirates. ${ }^{3}$ Endodontic department, Al Ain Dental Centre, Seha Ambulatory Healthcare Services, Al Ain, Abu Dhabi, United Arab Emirates. ${ }^{4}$ Dental department, Mediclinic, Dubai, United Arab Emirates. 
Received: 26 March 2020 Accepted: 1 October 2020

Published online: 07 October 2020

\section{References}

1. Weine FS, Healey HJ, Gerstein H, Evanson L. Canal configuration in the mesiobuccal root of the maxillary first molar and its endodontic significance. J Endod. 1969;38:4.

2. Hartwell G, Appelstein CM, Lyons WW, Guzek ME. The incidence of four canals in maxillary first molars: a clinical determination. J Am Dent Assoc. 2007;138(10):1344-6.

3. Karabucak B, Bunes A, Chehoud C, Kohli MR, Setzer F. Prevalence of apical periodontitis in Endodontically treated premolars and molars with Untreated Canal: a cone-beam computed tomography study. J Endod. 2016;42(4):538-41.

4. Siqueira JF. Aetiology of root canal treatment failure: why well-treated teeth can fail. Int Endod J2nd ed. John Wiley \& Sons, Ltd. 2001;34(1):1-10.

5. Wolcott J, Ishley D, Kennedy W, Johnson S, Minnich S, Meyers J. A 5 yr clinical investigation of second mesiobuccal canals in endodontically treated and retreated maxillary molars. J Endod. 2005;31(4):262-4.

6. Alavi AM, Opasanon A, Ng Y-L, Gulabivala K. Root and canal morphology of Thai maxillary molars. Int Endod JJohn Wiley \& Sons, Ltd. 2002;35(5):478-85.

7. Neaverth EJ, Kotler LM, Kaltenbach RF. Clinical investigation (in vivo) of endodontically treated maxillary first molars. J Endod. 1987;13(10):506-12.

8. Kulild JC, Peters DD. Incidence and configuration of canal systems in the mesiobuccal root of maxillary first and second molars. J Endod. 1990;16(7): 311-7.

9. Stropko JJ. Canal morphology of maxillary molars: clinical observations of canal configurations. J Endod. 1999;25(6):446-50.

10. Zhang $\mathrm{R}$, Yang $H$, Yu X, Wang $H, H u T$, Dummer PMH. Use of CBCT to identify the morphology of maxillary permanent molar teeth in a Chinese subpopulation. Int Endod J. 2011;44(2):162-9.

11. Zheng Q-H, Wang Y, Zhou X-D, Wang Q, Zheng G-N, Huang D-M. A conebeam computed tomography study of maxillary first permanent molar root and canal morphology in a Chinese population. J Endod. 2010;36(9):1480-4.

12. Hoen MM, Pink FE. Contemporary endodontic retreatments: an analysis based on clinical treatment findings. J Endod. 2002;28(12):834-6.

13. Cantatore G, Berutti E, Castellucci A. Missed anatomy: frequency and clinical impact. Endod TopJohn Wiley \& Sons, Ltd. 2006;15(1):3-31.

14. Cleghorn BM, Christie WH, Dong CCS. Root and root canal morphology of the human permanent maxillary first molar: a literature review. J Endod. 2006;32(9):813-21.

15. Neelakantan P, Subbarao C, Subbarao CV. Comparative evaluation of modified canal staining and clearing technique, cone-beam computed tomography, peripheral quantitative computed tomography, spiral computed tomography, and plain and contrast medium-enhanced digital radiography in studying root canal morphology. J Endod. 2010; 36(9):1547-51.

16. Blattner TC, George N, Lee CC, Kumar V, Yelton CDJ. Efficacy of cone-beam computed tomography as a modality to accurately identify the presence of second mesiobuccal canals in maxillary first and second molars: a pilot study. J Endod. 2010;36(5):867-70.

17. Domark JD, Hatton JF, Benison RP, Hildebolt CF. An ex vivo comparison of digital radiography and cone-beam and micro computed tomography in the detection of the number of canals in the mesiobuccal roots of maxillary molars. J Endod. 2013:39(7):901-5.

18. Martins JNR, Marques D, Mata A, Caramês J. Root and root canal morphology of the permanent dentition in a Caucasian population: a conebeam computed tomography study. Int Endod J1st ed. John Wiley \& Sons, Ltd. 2017;50(11):1013-26.

19. Pérez-Heredia M, Ferrer-Luque CM, Bravo M, Castelo-Baz P, Ruíz-Piñón M, Baca P. Cone-beam computed tomographic study of root anatomy and canal configuration of molars in a Spanish population. J Endod. 2017;43(9): 1511-6.

20. de AR RAG, Grazziotin-Soares R, Barletta FB, VRC F, CRW M. Second canal in mesiobuccal root of maxillary molars is correlated with root third and patient age: a cone-beam computed tomographic study. J Endod. 2013; 39(5):588-92.

21. Plotino G, Tocci L, Grande NM, Testarelli L, Messineo D, Ciotti M, et al. Symmetry of root and root canal morphology of maxillary and mandibular molars in a white population: a cone-beam computed tomography study in vivo. J Endod. 2013;39(12):1545-8.
22. Ghobashy AM, Nagy MM, Bayoumi AA. Evaluation of root and canal morphology of maxillary permanent molars in an Egyptian population by cone-beam computed tomography. J Endod. 2017:43(7):1089-92.

23. Guo J, Vahidnia A, Sedghizadeh P, Enciso R. Evaluation of root and canal morphology of maxillary permanent first molars in a north American population by cone-beam computed tomography. J Endod. 2014;40(5):6359.

24. Khademi A, Zamani Naser A, Bahreinian Z, Mehdizadeh M, Najarian M, Khazaei S. Root morphology and canal configuration of first and second maxillary molars in a selected Iranian population: a cone-beam computed tomography evaluation. Iran Endod J. 2017;12(3):288-92.

25. Kim Y, Lee S-J, Woo J. Morphology of maxillary first and second molars analyzed by cone-beam computed tomography in a korean population: variations in the number of roots and canals and the incidence of fusion. J Endod. 2012:38(8):1063-8.

26. Aktan AM, Yildirim C, Culha E, Demir E, Ertugrul CM. Detection of second Mesiobuccal canals in maxillary first molars using a new angle of cone beam computed tomography. Iran J Radiol Kowsar. 2016;13(4):e31155.

27. Al-Shehri. Root and canal configuration of the maxillary first molar in a Saudi subpopulation: a cone-beam computed tomography study. Saudi Endod JMedknow Publications. 2017:7(2):69.

28. Brühschwein A, Klever J, Hoffmann A-S, Huber D, Kaufmann E, Reese S, et al Free DICOM-viewers for veterinary medicine : survey and comparison of functionality and user-friendliness of medical imaging PACS-DICOM-viewer freeware for specific use in veterinary medicine practices. J Digit ImagingSpringer International Publishing. 2019;27(4):470-10.

29. Vertucci FJ. Root canal anatomy of the human permanent teeth. Oral Surg Oral Med Oral Pathol. 1984;58(5):589-99.

30. Weine FS, Healey HJ, Gerstein H, Evanson L. Canal configuration in the mesiobuccal root of the maxillary first molar and its endodontic significance. Oral Surg Oral Med Oral Pathol. 1969;28(3):419-25.

31. Ball RL, Barbizam JV, Cohenca N. Intraoperative endodontic applications of cone-beam computed tomography. J Endod. 2013 Apr;39(4):548-57.

32. Salem S, Ibrahim SM, ISSN AAASDS. Prevalence of second Mesio-Buccal Canal in maxillary first and second molars in Egyptian population using CBCT (a cross-sectional study); 2018.

33. Mashyakhy M, Chourasia HR, Jabali A, Almutairi A, Gambarini G. Analysis of fused rooted maxillary first and second molars with merged and C-shaped canal configurations: prevalence, characteristics, and correlations in a Saudi Arabian population. J Endod. 2019:1:1-10.

34. Neelakantan P, Subbarao C, Ahuja R, Subbarao CV, Gutmann JL. Conebeam computed tomography study of root and canal morphology of maxillary first and second molars in an Indian population. J Endod. 2010;36(10):1622-7.

35. Martins JNR, Alkhawas M-BAM, Altaki Z, Bellardini G, Berti L, Boveda C, et al. Worldwide analyses of maxillary first molar second Mesiobuccal prevalence: a multicenter cone-beam computed tomographic study. J Endod. 2018; 44(11):1641

36. Oi T, Saka H, Ide Y. Three-dimensional observation of pulp cavities in the maxillary first premolar tooth using micro-CT. Int Endod JJohn Wiley \& Sons, Ltd. 2004;37(1):46-51.

37. Hülsmann M. Prevention and management of problems during root canal treatment-a problem-based approach to root canal treatment part I. ENDO (Lond Engl). 2016;10(3):127-40.

38. Peters $\mathrm{O}$. Current challenges and concepts in the preparation of root canal systems: a review. J Endod. 2004;30(8):559-67.

39. Kottoor J, Velmurugan N, Surendran S. Endodontic Management of a Maxillary First Molar with eight root canal systems evaluated using conebeam computed tomography scanning: a case report. J Endod. 2011;37(5): $715-9$.

40. Albuquerque D, Kottoor J, Hammo M. Endodontic and clinical considerations in the management of variable anatomy in mandibular premolars: a literature review. Biomed Res Int1st ed. Hindawi. 2014;2014(6): 512574-11.

41. Sahoo HS, Kurinji Amalavathy R, Pavani D. A case report on endodontic Management of a Rare Vertucci Type III maxillary canine. Case Rep DentHindawi. 2019;2019:4154067-4

42. Eder A, Kantor M, Nell A, Moser T, Gahleitner A, Schedle A, et al. Root canal system in the mesiobuccal root of the maxillary first molar: an in vitro comparison study of computed tomography and histology. Dentomaxillofac Radiol. 2006;35(3):175-7. 
43. Bauman R, Scarfe W, Clark S, Morelli J, Scheetz J, Farman A. Ex vivo detection of mesiobuccal canals in maxillary molars using CBCT at four different isotropic voxel dimensions. Int Endod J. 2011;44(8): 752-8.

44. Ji Y, Wen S, Liu S, Zhu M, Yao M, Wang T, et al. Could cone-beam computed tomography demonstrate the lateral accessory canals? BMC Oral Health. 2017;17(1):142.

\section{Publisher's Note}

Springer Nature remains neutral with regard to jurisdictional claims in published maps and institutional affiliations.

Ready to submit your research? Choose BMC and benefit from:

- fast, convenient online submission

- thorough peer review by experienced researchers in your field

- rapid publication on acceptance

- support for research data, including large and complex data types

- gold Open Access which fosters wider collaboration and increased citations

- maximum visibility for your research: over $100 \mathrm{M}$ website views per year

At BMC, research is always in progress. 